\title{
Encuesta Nacional de Opinión Pública del ICHEM: Análisis Longitudinal de Resultados 2009 - 2012
}

Jorge Morales Jacob ${ }^{26}$

Fecha de Recepción: 7/07/2012

Fecha de Aceptación: 20/08/20I2

Resumen

Desde el año 2008, el Instituto Chileno de Estudios Municipales de la Universidad Autónoma de Chile (ICHEM) ha desarrollado, como una de sus principales actividades, una encuesta que tiene como especificidad conocer la percepción de los habitantes de distintas comunas de Chile sobre sus municipios y temas locales que les conciernen. A partir de allí, se han elaborado preguntas que han estado presentes en todas las versiones de esta medición.

En este artículo, se realiza una mirada longitudinal de los resultados que arroja la Encuesta para el período 2009 - 2012, con respecto a temas que han sido relevantes en la agenda nacional y local. Entre los resultados expuestos, el análisis muestra, no sin variaciones, que la población percibe la Delincuencia como el principal problema que la afecta, manteniéndose la tendencia. En cambio, el problema de la Atención de Salud primaria emerge significativamente como problema central en los municipios recién a partir de los últimos tres años (2010 20I2). Algo similar sucede con la Educación, que solo a partir de los últimos dos años (20II - 2012), cobra mayor relevancia en la población encuestada a nivel comunal.

Palabras clave: Percepción ciudadana, opinión pública local, municipios, problemas locales, educación, salud, seguridad ciudadana

Abstract

Since 2009, the Instituto Chileno de Estudios Municipales - Chilean Instituto of Municipal Studies has carried out, as one of its main activities, a poll whose purpose is to know how the residents of local communities in Chile perceive their municipalities and the topics they are in charge of.Thereupon, it has devised questions that have been included in all of the versions of these samplings.

Th present article presents a longitudinal look of the results that have come up from its main poll for the 2009-20I I term of those topics that have been relevant in the national and local agenda. Among those results, the analysis shows, with different variations, that the population perceives delinquency as the main problem that affects them. In this item, the tendency has mantained. On the other hand, the problem of Primary Health Attention has emerged recently as a central dilemma in municipalities (20I0-20I2). Something similar occurs with Education. Only since the two last years (20II-20I2) it has become an important issue among the people surveyed in local areas.

Key Words: Population perception, local public opinion, municipalities, local problems, education, health, public safety.

26.- Sociologo (Universidad de Chile) y Profesor de Enseñanza Media en Matemática y Estadística (ARCIS), cursa el IV Semestre del Magister de Gestión Pública y Desarrollo Regional y Local (Universidad de Los Lagos). Es Investigador del Instituto Chileno de Estudios Municipales. Correo: jmorales@ichem.cl 


\section{Resumo}

Desde o ano 2008, o Instituto Chileno de Estudos Municipais da Universidade Autônoma de Chile (ICHEM) tem desenvolvido, como uma das suas principais atividades, uma pesquisa que tem como especificidade conhecer a percepção dos habitantes de distintas comunas de Chile sobre seus municípios e temas locais que corresponde a eles. A partir de este momento, se elaboraram perguntas que estão presentes em todas as versões de esta medição

Em este artigo, se realiza uma mirada longitudinal dos resultados que entrega a Pesquisa para o período 2009 - 2012, com respeito a temas que foram relevantes na agenda nacional e local. Entre os resultados mostrados, a análise mostra sem variações que a população percebe a Delinquência como o principal problema que afeta, mantendo a tendência. Em troca, o problema da Atenção da Saúde primaria emerge significativamente como problema central nos municípios recém a partir dos últimos três anos (20l0 - 20I2).Algo parecido acontece com a Educação, que somente a partir dos últimos dois anos (20II - 20I2), cobra maior relevância na população pesquisada em nível de comuna.

Palavras-Chave: Percepção dos cidadãos, opinião pública local, municípios, problemas locais, educação, saúde, segurança dos cidadãos.

\section{Introducción}

El Instituto Chileno de Estudios Municipales de la Universidad Autónoma de Chile (ICHEM), a través su Área de Estudios, ha realizado, desde su creación, diversos estudios sobre variadas temáticas municipales. ${ }^{27}$ Uno de los estudios más importantes que ha realizado es la Encuesta Nacional de Opinión Pública (en adelante ENOP) que el ICHEM aplica, a nivel nacional y de manera periódica, desde el año 2009. Esta encuesta se diseñó con el objetivo de conocer y registrar las percepciones que los propios habitantes de las comunas tienen sobre diversos temas a nivel local, a saber: problemas, autoridades y mecanismos de participación en sus territorios comunales; aplicando para ello una mirada longitudina ${ }^{28}$ a cada fenómeno estudiado.

Con los años, la encuesta ha sido modificada para abordar temáticas que van siendo contingentes año a año, pero siempre conservando los temas nucleares con los que se estructuró en un comienzo.

El presente artículo aborda, desde un análisis longitudinal, los resultados de sus aplicaciones, es decir, las percepciones de los encuestados acerca de los principales temas revisados en la encuesta a lo largo de los años, temas que resultan susceptibles de ser revisados en el actual escenario municipal en Chile.

El objetivo de trazar una línea longitudinal es constatar si han existido cambios significativos en las variables estudiadas para así observar la posible presencia de una evolución o modificación en las demandas para los municipios.

\section{7.- Puede descargar los estudios de los años 201 I y 2012 en www.ichem.cl}

28.- Se entiende por análisis longitudinal de resultados aquel que observa el comportamiento, en este caso, de una serie de mediciones a muestras representativas de una misma población, a lo largo del tiempo - Diseño de Tendencias. (Tipos de encuestas considerando la dimensión temporal,Vidal Díaz de Rada, Universidad Pública de Navarra. Departamento de Sociología Campus de Arrosadía s/n. 31006 Pamplona- Papers 86, 2007) 


\section{El Problema}

Los municipios no son solo constructos político administrativos, sustentados en andamiajes jurídicos, que deben cumplir con tareas específicas en territorios determinados; no solo administran bienes y servicios para la comunidad. La visión actual de municipio ve al gobierno comunal como una instancia capaz de articular políticas públicas respondiendo a los intereses y necesidades de la comunidad y entendiendo que estos no son estáticos, sino que varían durante el tiempo (Delamaza y Flores, 2009; Durston, 1999). Para lograr estos objetivos es necesario conocer cuáles son los intereses y necesidades de las personas y, lo más relevante, saber cómo cambian y evolucionan.

Los estudios sobre percepción de temas de opinión pública en Chile (encuestas CEP, CERC, Adimark, por ejemplo) han utilizado un enfoque transversal para analizar las percepciones a nivel local.Y, muchas veces, sustentados en una visión de lo local o comunal como instancia jurídica - el municipio- antes que como un territorio articulado en una compleja trama de dimensiones que lo constituyen como tal.

A la luz de la limitación de dichos estudios, se ha considerado necesario desde el ICHEM tener una visión diferente sobre el fenómeno de la percepción de los gobiernos locales. Por eso, la ENOP ha sido concebida como una encuesta aplicable longitudinalmente, la que, a través del tiempo, se ha modificado y ampliado para abordar los temas originales de la encuesta en una clave más local. A la vez, con los años se le han introducido también nuevas temáticas; dicha inclusión ha querido responder a las inquietudes y temas que la propia ciudadanía ha puesto en la agenda nacional.

\section{Metodología e Historia de la ENOP}

Desde sus inicios, la Encuesta Nacional de Opinión Pública (ENOP) fue conceptualizada como una encuesta que formaba parte de una investigación cuantitativa de tipo exploratorio descriptiva, de carácter longitudinal. En tal sentido, se entendió, a manera de hipótesis, que la percepción sobre los temas que conformaban la encuesta variaría de año en año, dependiendo de las contingencias que experimentara la agenda pública. También se asumió que, por esos mismos motivos, la ENOP podría experimentar algún cambio, sin que ello significase eliminar los temas que la constituyeron en un inicio. Así, para comprender la metodología de la ENOP resulta conveniente revisar su historia y evolución.

\section{Historia y Evolución de la ENOP}

Originalmente, la ENOP se diseñó en base a las distintas encuestas que en esos momentos se realizaban en Chile en torno a la temática municipal y desde la misma experiencia de estudios del ICHEM, considerando algunos aspectos que tenían que ver con el entorno local a nivel ciudadano. El enfoque de esta primera versión de la ENOP se centraba en la percepción de los aspectos jurídico administrativos de los municipios en Chile, dando cierta relevancia a los temas locales.

La primera versión de la ENOP data de 2009. Sus contenidos se centraban en cinco grandes temas, incluidos en sendos módulos y divididos en 24 preguntas. Ese mismo año se aplicó a una muestra de I.487 personas, de distintas comunas de Chile. Los temas que la encuesta tocaba en ese entonces versaban sobre el conocimiento y evaluación de:
a) La institucionalidad municipal
b) Los problemas de la comuna
c) Los temas que debieran tratar las autoridades municipales
d) Instrumentos de participación social
e) Seguridad ciudadana 
El 2010 se decidió incorporar un módulo de educación, que incluía preguntas sobre la evaluación de la educación municipal. Esto permitió acercar la encuesta a la realidad cotidiana de las personas de diferentes comunas. Ese año la ENOP se aplicó a I.33I personas, de diferentes comunas del país. El 201 I se incorporaron dos nuevos temas, a saber: la representatividad política de los gobernantes comunales, es decir, si la comunidad se sentía o no representada por sus autoridades. El segundo tema fue el tipo de liderazgo que las personas preferían para sus comunas. Estas nuevas incorporaciones se entendieron como elementos de percepción local y no como variables provenientes desde el marco jurídico administrativo. Ese año la encuesta se aplicó a una muestra de I.242 personas de distintas comunas del país.

Finalmente, el 2012 se incorporó un cuarto tema nuevo, la gestión del riesgo. Desde este tema se pudo visualizar cómo las personas evaluaban a sus gobernantes en su capacidad de respuesta ante catástrofes o desastres naturales. Lógicamente, la incorporación de esta nueva variable obedeció a los acontecimientos que experimentó Chile desde el terremoto y maremoto el 27 de febrero de 2010 en materia de desastres naturales. Este año la encuesta se aplicó a I.349 personas, de distintas partes del país, con muestras que han sido representativas de las 15 regiones de Chile. La encuesta se ha aplicado siempre bajo la modalidad cara a cara y ha tenido un error muestral que nunca ha superado el $3 \%$.

\section{I Temas Considerados para este artículo}

Para la redacción de este artículo, se decidió trabajar con aquellos temas que tuvieran mayor continuidad a lo largo de la historia de aplicación de la encuesta, es decir, que fueran susceptibles de ser considerados como utilizables en un análisis longitudinal.Así, los temas considerados para la entrega de sus resultados fueron el conocimiento y evaluación de la institucionalidad municipal, los problemas de la comuna y los temas que debieran tratar las autoridades municipales.

\section{Resultados}

Los siguientes resultados están divididos de acuerdo a los temas considerados como relevantes para este artículo. En cada presentación de resultados se hace un breve análisis descriptivo respecto de las variaciones experimentadas a lo largo de los años.

Conocimiento y Evaluación sobre la Institucionalidad Municipal: ¿Quién administra qué? ¿Quién debería administrar qué?

El siguiente gráfico da cuenta del resultado obtenido acerca de la educación pública:

Gráfico $N^{\circ}$ I. Conocimiento a nivel local sobre quién es responsable de la Educación pública

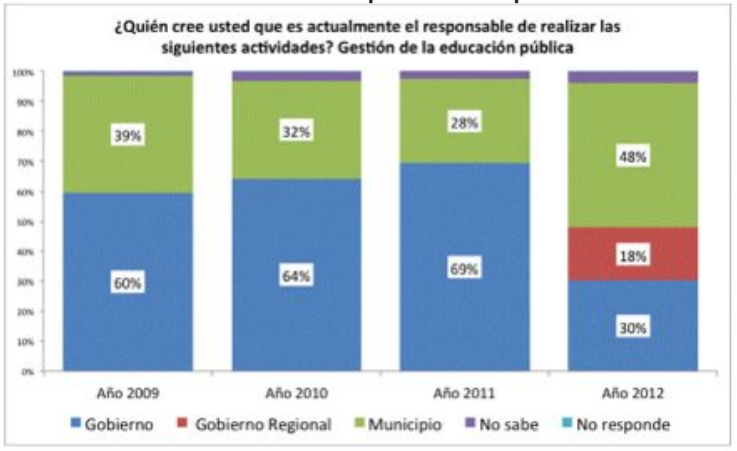

Fuente: Encuesta Nacional de Opinión Pública, ICHEM.Años 2009 - 2012. 
La serie de datos 2009 - 2012 de la ENOP incluye preguntas con respecto al conocimiento que la población tiene sobre el ejercicio de ciertas responsabilidades. En este sentido, un primer aspecto importante a destacar dice relación con la Educación pública. Entre los años 2009 y $201 \mathrm{I}$ la opinión de la población señala en forma creciente que es el Gobierno el responsable de dicho tema, pasando de un 60 por ciento a un 69 por ciento. Otra forma de mirar esta serie, e interesante de resaltar, es que decrece la opinión de los chilenos y chilenas de que son los municipios los responsables de la educación pública, pasando de un 39 por ciento a un 28 por ciento. Sin embargo, observando los datos para la encuesta 2012 , la tendencia se revierte drásticamente: los municipios son los encargados actuales de la educación pública, según el 48 por ciento de la opinión pública. En contraposición, sólo un 30 por ciento señala que ésta es de responsabilidad del Gobierno central.

El siguiente gráfico da cuenta de los resultados sobre la salud primaria:

Gráfico $N^{\circ}$ 2. Conocimiento a nivel local sobre quién es responsable de la Atención de Salud Primaria

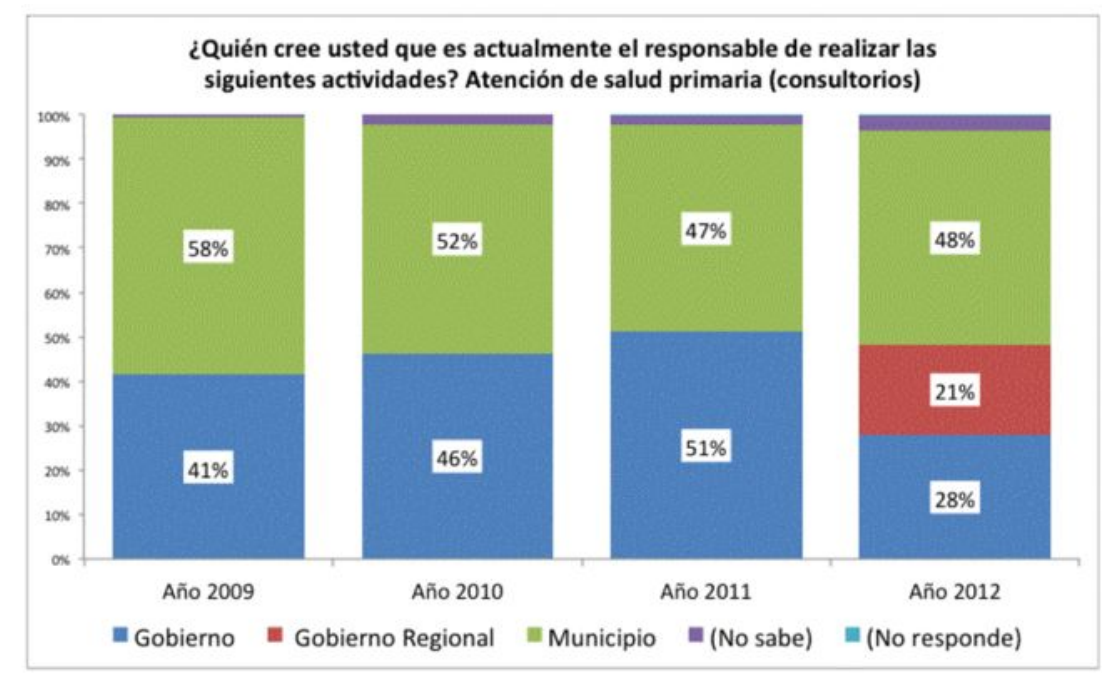

Fuente: Encuesta Nacional de Opinión Pública, ICHEM.Años 2009 - 2012.

Con respecto a la Atención de Salud Primaria, la tendencia indica que una parte importante de la población opina que son los municipios los encargados de administrarla y gestionarla. Para la encuesta de 2009 se encuentra el peak, donde un 58 por ciento de la población adhiere a esta opción, mientras que un $4 \mathrm{I}$ por ciento indica que la Atención de Salud Primaria es de responsabilidad del Gobierno.Aunque es posible observar que para los años posteriores hay una disminución en la proporción de opiniones que entregan la responsabilidad a los municipios, llegando al 48 por ciento el 2012, la tendencia de los últimos tres años indica que la población adjudica esta responsabilidad en forma similar tanto a los municipios como al Gobierno.

El gráfico siguiente muestra la variación acerca de la seguridad pública: 
Gráfico $N^{\circ}$ 3. Conocimiento a nivel local sobre quién es responsable de la Seguridad pública

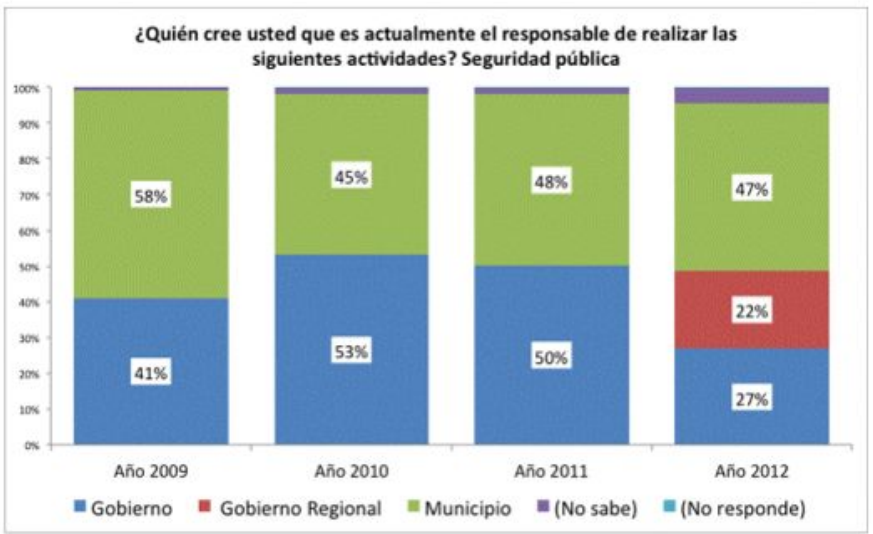

Fuente: Encuesta Nacional de Opinión Pública, ICHEM.Años 2009 - 2012.

En materia de Seguridad Pública, los datos de la serie 2009-2012 indican que la opinión pública asigna responsabilidades tanto a los municipios como al Gobierno. Si bien es cierto que la encuesta de 2009 da cuenta de una mayor proporcionalidad en los municipios, los años siguientes manifiestan una tendencia a la baja, siendo el último dato, 47 por ciento, el que refleja la encuesta del presente año.

A manera de contrapunto, los gráficos siguientes muestran la opinión de la población acerca de qué organismo administraría mejor ciertos ámbitos. Se trata, pues, de preferencias por una administración centralizada o descentralizada.

El gráfico siguiente muestra quién debería administrar la Educación pública, percepciones recogidas durante el período 2009 - 2012:

Gráfico $N^{\circ}$ 4. Preferencias acerca de la gestión de la Educación pública

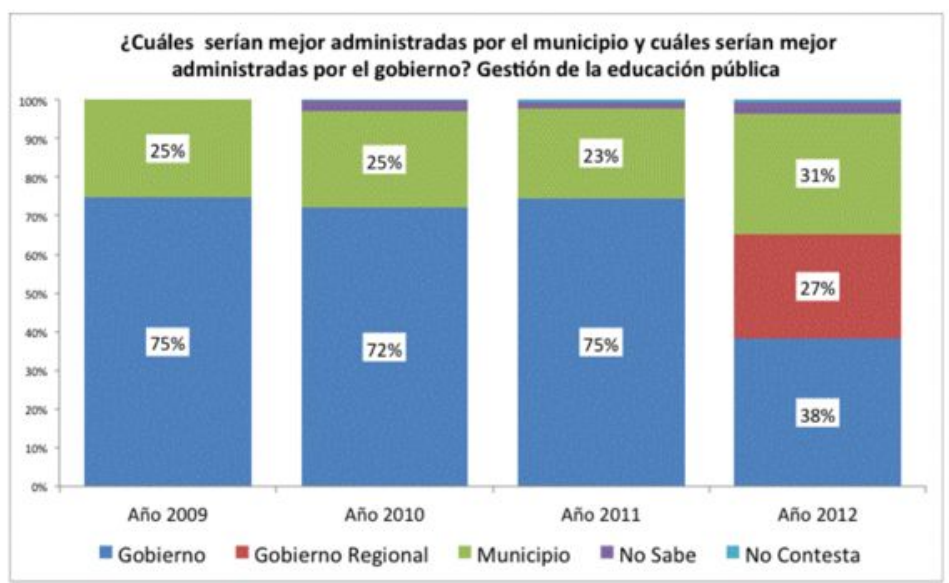

Fuente: Encuesta Nacional de Opinión Pública, ICHEM.Años 2009 - 2012.

En este ámbito puede observarse que la mayor parte de la población es partidaria de una administración centralizada de la Educación pública, vale decir, por parte del Gobierno. 
Si bien entre 2009 y 201 I los datos permanecen estables, en 2012 destaca que existe una mayor proporción de opiniones partidarias de una educación pública administrada y gestionada desde los gobiernos locales.

El gráfico siguiente muestra la percepción respecto de quién debería administrar la Atención de Salud Primaria:

Gráfico N 5. Preferencias acerca de la Gestión de Atención de Salud Primaria

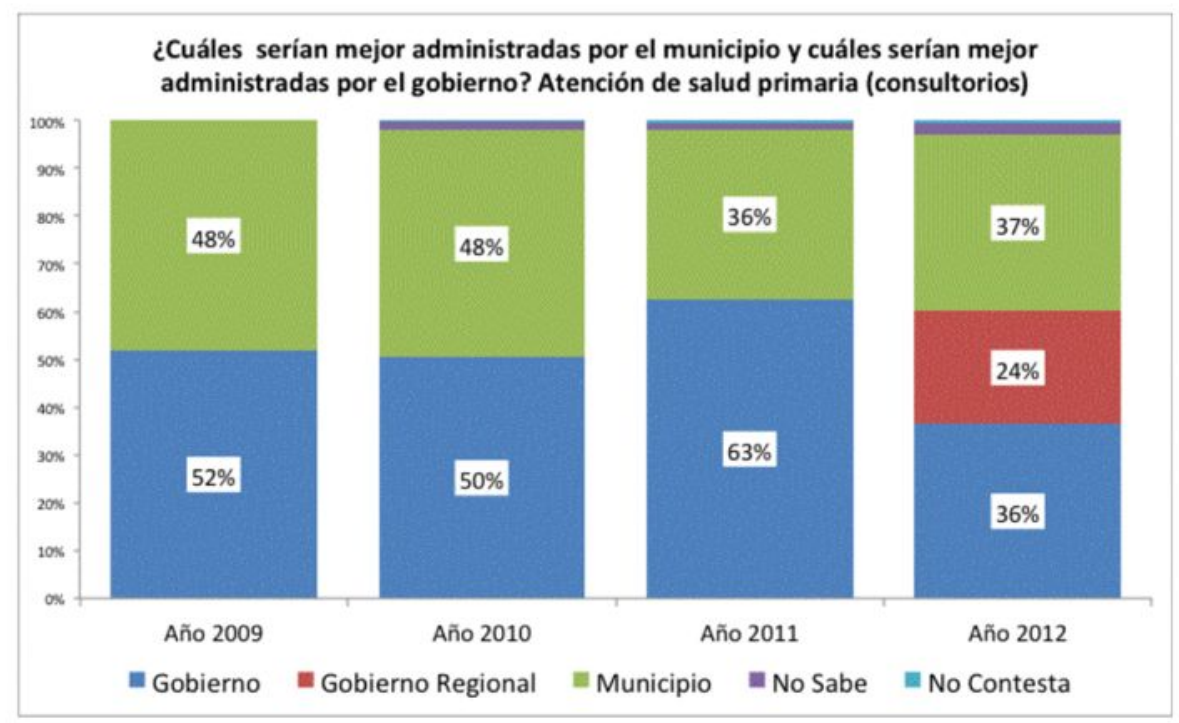

Fuente: Encuesta Nacional de Opinión Pública, ICHEM. Años 2009 - 2012.

Con respecto a la Atención de Salud Primaria -que corresponde a la figura de los consultorios- los datos de la serie 2009 - 2012 presentan cambios mucho más marcados que los de Educación. En este sentido, los años 2009 y 2010 muestran que las opiniones sobre una mejor administración se encuentran similarmente divididas entre Gobierno y Municipio. Sin embargo, en la recolección de datos de los años 201 l y 2012 se puede apreciar una baja significativa del porcentaje de población que opina que es en los gobiernos locales donde se puede administrar de mejor forma la Salud primaria: mientras que durante el 2009 y 2010 un 48 por ciento de la opinión pública se pronunciaba por esta alternativa, el $201 \mathrm{I}$ y 2012 esta proporción disminuye a un 36 y 37 por ciento, respectivamente.

El gráfico siguiente muestra la percepción respecto de quién debería administrar la Seguridad Pública: 
Gráfico $N^{\circ}$ 6. Preferencias acerca de la gestión de la Seguridad Pública

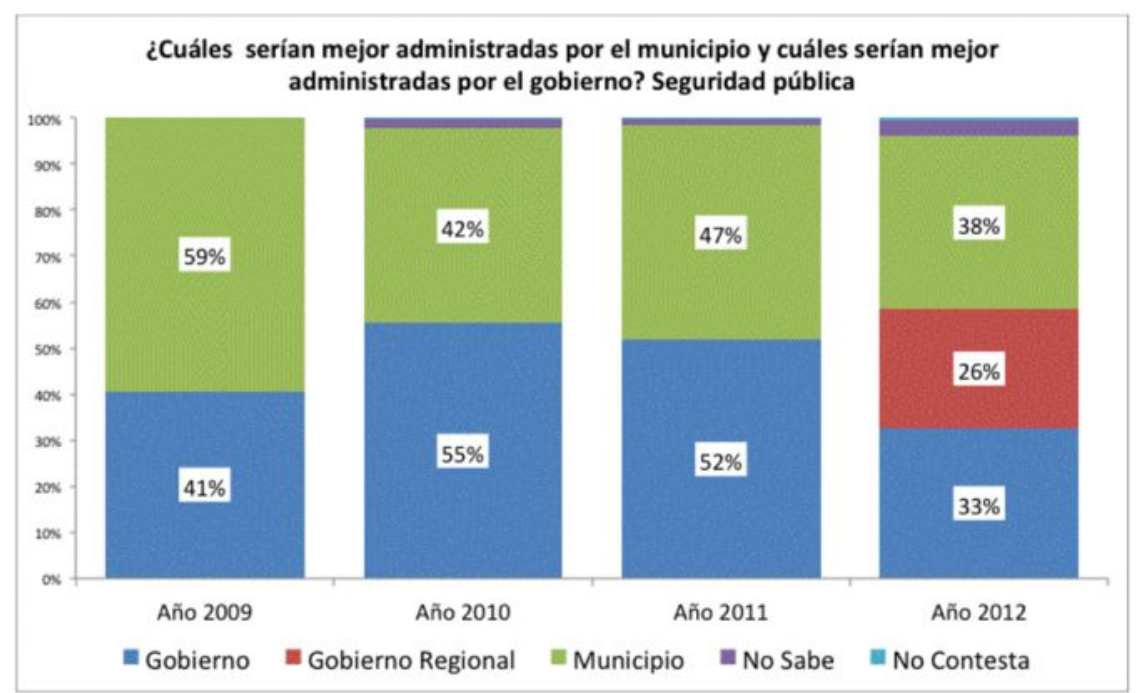

Fuente: Encuesta Nacional de Opinión Pública, ICHEM.Años 2009 - 2012.

En el ámbito de la Seguridad Pública, los datos de la serie 2009 - 2012 muestran una tendencia que progresa hacia un deseo centralizador, aunque no del todo muy claro. En los cuatro años de la serie, tan sólo uno, el inicial, indica que la población cree que es el Municipio el organismo que mejor administraría la Seguridad Pública. Sin embargo, en las aplicaciones posteriores de la encuesta, los datos revelan que esta proporcionalidad se revierte, teniendo para el 2012 a poco más de un tercio de la población (38 por ciento) que sigue manteniendo dicha opinión. Como se puede apreciar en el gráfico, los últimos tres años evidencian que el grueso de las opiniones adhiere a que sería el Gobierno quien pudiese administrar mejor la Seguridad Pública. 
Conocimiento y Evaluación de los Problemas de la Comuna

El gráfico correspondiente a este tema es el siguiente:

Gráfico $N^{\circ} 7$. Percepciones acerca de los principales problemas que afectan a la comuna

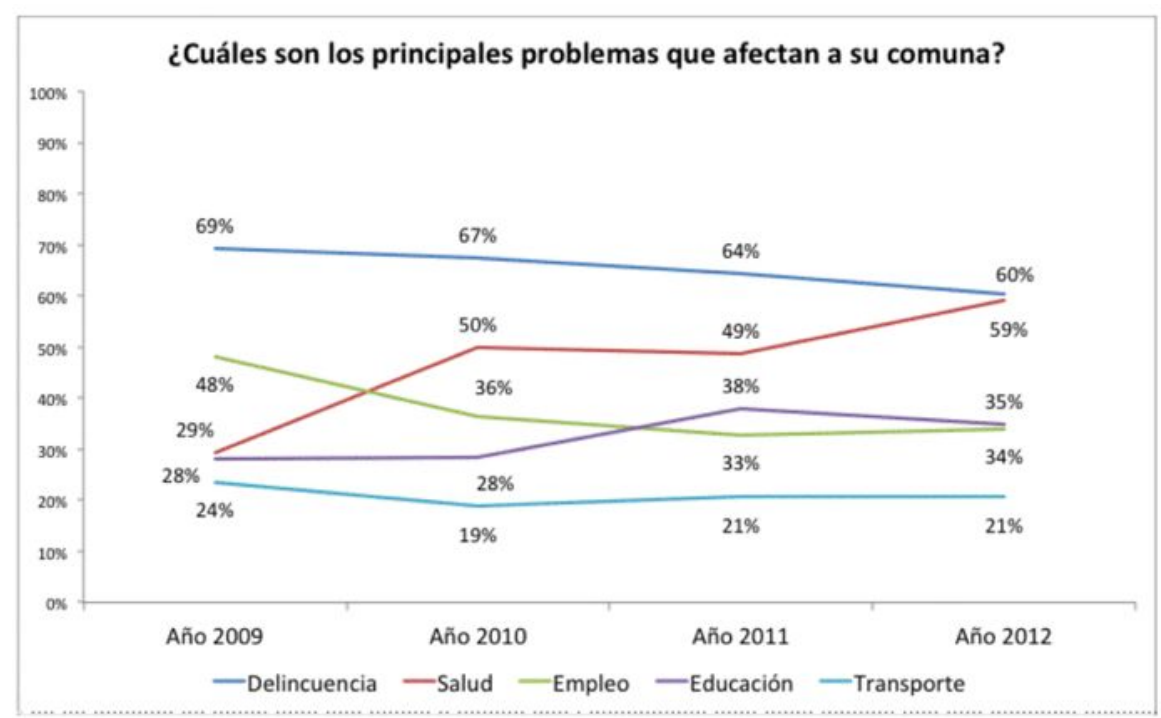

Fuente: Encuesta Nacional de Opinión Pública, ICHEM. Años 2009 - 2012.

La serie de datos 2009 - 2012 permite evidenciar que el problema más destacado en el período dice relación con la Delincuencia. En este sentido, los cuatro años de aplicación de la encuesta permiten observar que éste corresponde al tema con mayor preponderancia, aun cuando también se observa una baja sistemática desde un 69 a un 60 por ciento, a lo largo de los años.

Un segundo elemento importante de destacar en relación a los principales problemas que afectan a la comuna, corresponde al tema Salud. Como se puede observar, el tema emerge sistemáticamente en la percepción de la población: si para 2009 sólo un 29 por ciento de los encuestados lo asumía como relevante, los datos del 2012 lo posicionan como el segundo problema más importante, con un 59 por ciento y sólo I punto porcentual por debajo que la Delincuencia.

Con respecto a la opinión sobre Empleo, en cuanto problema que afecta a la comuna, se observa una baja no despreciable. Tomando la serie 2009 - 2012, se puede apreciar que inicia como el segundo mayor problema percibido por la población, pero que sufre una caída hasta ubicarse en el tercer lugar: baja de un 48 a un 34 por ciento. Esta situación contrasta, aunque no en magnitud, con lo ocurrido en el caso de la Educación: mientras que en 2009 se presenta como el cuarto problema que afecta a la comuna, en el transcurso del período de evaluación logra posicionarse como el tercero, alcanzando un 35 
por ciento de adhesión en la población.

Finalmente, es preciso señalar que el problema de Transporte mantiene su lugar como el menos priorizado por la población: de 2009 a 2012 pasa de un 24 a un 21 por ciento, significativamente por debajo de los recientemente descritos.

Conocimiento y Evaluación de los Temas que Debieran Tratar las Autoridades Municipales

El gráfico que corresponde a este tema es el siguiente:

Gráfico $N^{\circ}$ 8. Percepciones acerca de los temas más importantes a tratar por el municipio

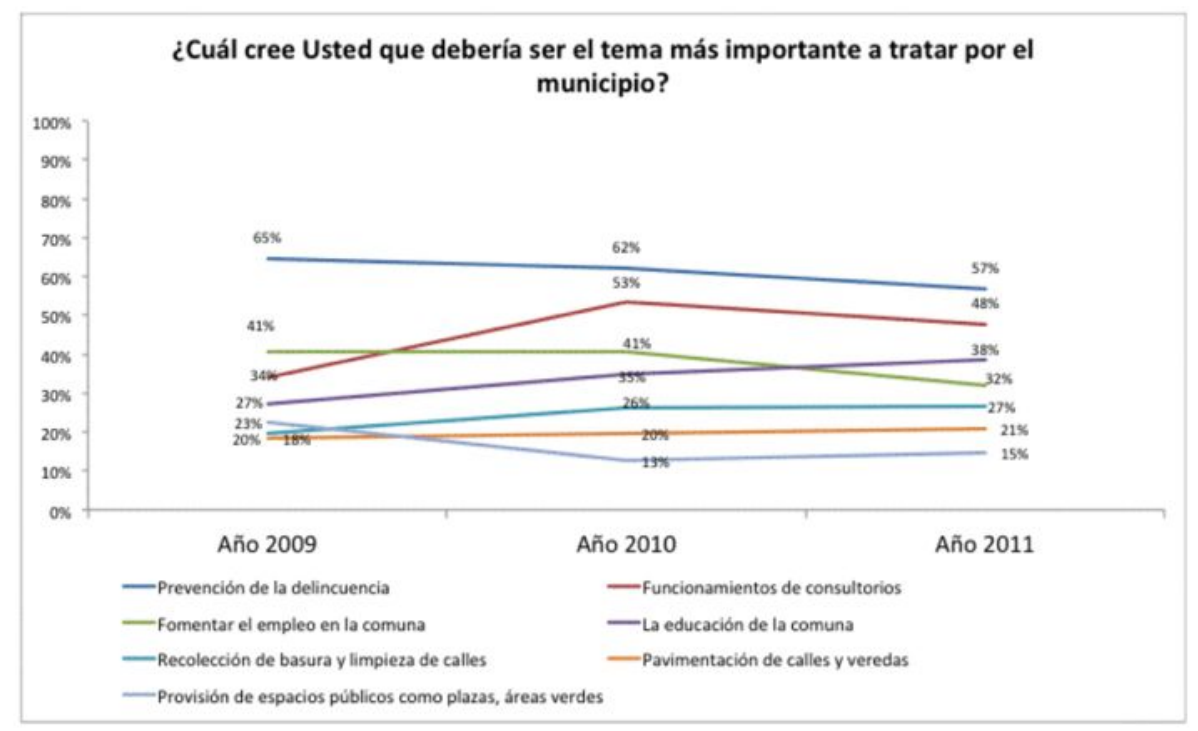

Fuente: Encuesta Nacional de Opinión Pública, ICHEM.Años 2009 - 201 I.

La serie de datos 2009 - 2011 muestra información importante sobre los temas que, según la población, debiesen ser tratados por el municipio. En este sentido, lo más significativo y concordante con los datos anteriores corresponde a la Prevención de la Delincuencia: si bien es cierto que muestra una baja importante, del 65 por ciento en 2009 al 57 por ciento en $20 \mathrm{I}$ I, debe señalarse que continúa siendo un ámbito que se indica como el tópico de mayor relevancia a tratar por la administración comunal.

El segundo aspecto que la población prioriza para ser tratado por los municipios corresponde al funcionamiento de los consultorios de Atención de Salud Primaria. Es necesario destacar que en el período 2009 - 20 I I este tema ha sufrido una evolución en su posicionamiento, dado que para 2009 representaba el 34 por ciento de las preferencias de la población y ocupaba el tercer lugar; sin embargo, para el año $20 \mathrm{II}$ alcanza un 48 por ciento de adhesión y se ubica como el segundo tema más importante del cual los municipios se debieran ocupar. 
La Educación de la comuna corresponde a un tema que cobra relevancia en el período de datos 2009 - 20II. Como se puede apreciar en el gráfico que sigue a continuación, éste inicialmente obtiene un 27 por ciento de las preferencias, pero, para el último año de evaluación alcanza un 38 por ciento y se posiciona como el tercer problema más importante del cual los municipios se debieran ocupar.

En términos de relevancia, se puede indicar que el tema más afectado por las alzas de Funcionamiento de Consultorios y Educación de la comuna corresponde al tema de Fomento al Empleo. Esto porque si bien en 2009 emergía como el segundo tema más importante de la agenda municipal según la población, con un $4 \mathrm{I}$ por ciento de adhesión, en 20 I I descendió al cuarto puesto, bajando 9 puntos porcentuales y obteniendo un 32 por ciento de las preferencias.

\section{Conclusión}

Los resultados muestran tres fenómenos preponderantes que pueden vincularse con diferentes temas que han estado en la agenda pública en los últimos dos años, a saber: Educación, Salud y Delincuencia.

Educación

Como es conocido, los movimientos estudiantiles de los últimos dos años han influido a la ciudadanía en lo que a opiniones y propuestas se refiere. Es quizás por eso que la población visualiza el problema como una de las principales áreas en las que el municipio debe tomar cartas y como uno de los principales problemas de la comuna. También se entiende que es la influencia del movimiento estudiantil la que ha provocado que las personas crean que el Gobierno (a nivel nacional) es quien debe hacerse cargo de la educación pública, considerando que esta ha sido una de las más bulladas y difundidas propuestas de los estudiantes y de las ONG que trabajan en torno al tema. Lo curioso es que esto se contradice con la visión que tienen las mismas personas cuando señalan que el municipio sería el que mejor administraría los establecimientos educacionales públicos

Salud

Aparentemente, la Salud Primaria, vista como en crisis permanente, es otro de los temas que se ha perfilado como relevante en la opinión de las personas. Esto podría deberse a la constante aparición en medios de comunicación de las autoridades de salud nacional (especialmente el ministro) y los constantes reportajes y denuncias que se difunden tanto a través de medios como de redes sociales virtuales. Por eso, resulta lógico que las personas argumenten que la responsabilidad de la Atención de Salud Primaria ya no es sólo municipal, sino que se enmarca como un problema del Estado en su conjunto y que es el Gobierno el que debe participar en la solución de este problema, no solamente los municipios.

Seguridad Pública y Delincuencia

Uno de los temas centrales de la Derecha para ganar las elecciones presidenciales de 
2009 fue la "lucha contra la delincuencia". La falta de resultados que exhibir en ese ámbito, ampliamente difundida a través de medios de comunicación y redes sociales, sumada al reconocimiento de haber realizado una mala labor, le jugó en contra al actual Gobierno. Este puede ser un motivo posible de por qué las personas de distintas comunas estiman que el Gobierno nacional debiera tener mayor injerencia en el asunto. Ahora bien, este dato no tiene ningún tipo de relación con el hecho de que haya disminuido la percepción de la Delincuencia como uno de los principales problemas de la comuna; pero sí mantiene relación con la disminución de la percepción sobre la responsabilidad que le cabía al municipio sobre este tema.

De esta forma, se puede apreciar que los cambios en las percepciones recogidas se pueden vincular con fenómenos del acontecer nacional. Con el paso de los años se podrá incorporar nuevos temas al estudio longitudinal con base en la ENOP, cuando exista una cantidad suficiente de aplicaciones para poder establecer rangos comparativos adecuados.

Biblografía

Delamaza \& Flores. (2009). Estado actual y desafíos para la institucionalización de la Participación ciudadana en Chile. En Gestión Municipal Participativa: construyendo democracia cotidiana. Santiago de Chile: Lom Ediciones.

Durston, J. (1999). Construyendo Capital Social Comunitario. Revista de la CEPAL, Nº6, PP I03-I I8. Santiago de Chile: Cepal.

Flores, D., Pagliai, C., Lagarrigue, A. \& Leyton E. (20I I). Juegos en el poder municipal: una aproximación etnográfica. Revista Iberoamericana de Estudios Municipales, II (4), septiembre de 20II. 